\title{
Erratum: Shear viscosity of phase-separating polymer blends with viscous asymmetry [Phys. Rev. E. 63, 061403 (2001)]
}

\author{
H. S. Jeon and E. K. Hobbie \\ (Published 20 September 2001) \\ DOI: 10.1103/PhysRevE.64.049901 \\ PACS number(s): 83.80.Tc, 83.50.Ax, 47.55.Dz, 99.10.+g
}

In our paper, we describe rheo-optical measurements of the influence of domain morphology on the shear viscosity of phase-separating polymer blends with viscous asymmetry in the melt components. At the end of our paper, we offer a simple model for the effective shear viscosity $\left(\eta_{e f f}\right)$ of the mixtures over the range of shear rates $(\dot{\gamma})$ where the domain morphology is stringlike. Although the model was intended to be approximate and heuristic, it was also intended to be physical. We recently realized, however, that this is not the case, and we correct this oversight here. An expansion of the effective viscosity [Eq. (4) of our paper] as a function of $\phi_{d}$ (the volume fraction of the dispersed phase) in the dilute limit yields a leading-order correction proportional to $\phi_{d}^{1 / 2}$, implying that the intrinsic viscosity is infinite and the effective viscosity is not analytic. The origin of this problem is that the expression for $\phi_{d}$ derived in our paper assumes cylindrical symmetry, while the boundary condition for the continuity of tangential shear stress across an interface assumes planar symmetry. Although this boundary condition was used heuristically in an order-of-magnitude way, it is in fact incompatible with the expression derived for $\phi_{d}$.

The simple physical model that we intended to derive is one of layers in the shear-vorticity plane and was first suggested by van Oene [1]. Although the domains in such a model appear as sheets rather than strings, the alternate layers resemble strings when viewed in the shear-gradient plane, and a comparison of this model with experiment is worthwhile. The volume fraction of sheets is $\phi_{d} \sim D / l$, where $D$ is the mean thickness of a layer of the dispersed phase and $l$ is the mean separation between layers. Equation (3) and the analysis leading up to Eq. (4) in our paper are then valid, and Eq. (4) is replaced by

$$
\eta_{e f f} \sim \eta_{m} \dot{\gamma}_{m} h / \nu \approx \eta_{m}\left\{1+\phi_{d}\left(\frac{1}{\lambda}-1\right)\right\}^{-1}
$$

where $\lambda=\eta_{d} / \eta_{m}$. A small- $\phi_{d}$ expansion then yields $\eta_{e f f} / \eta_{m} \sim 1+(1-1 / \lambda) \phi_{d}+O\left(\phi_{d}^{2}\right)$, and the intrinsic viscosity is $1-1 / \lambda$. A comparison of the data with Eq. (1) is shown in Fig. 1, and yields $\lambda=3$ for $\phi_{d}<0.55$, and $\lambda=0.7$ for $\phi_{d}>0.55$, which can be compared with the experimental values of 2.67 and 0.37 , respectively. Although the values of $\lambda$ obtained from the new fit are virtually the same as those obtained previously, the shape of the curves are more consistent with the trends suggested by the data. An improvement on Eq. (1) that more closely corresponds to a stringlike morphology is desirable, but is beyond the scope of the present erratum. We regret this oversight and apologize for any inconvenience that it may have caused.

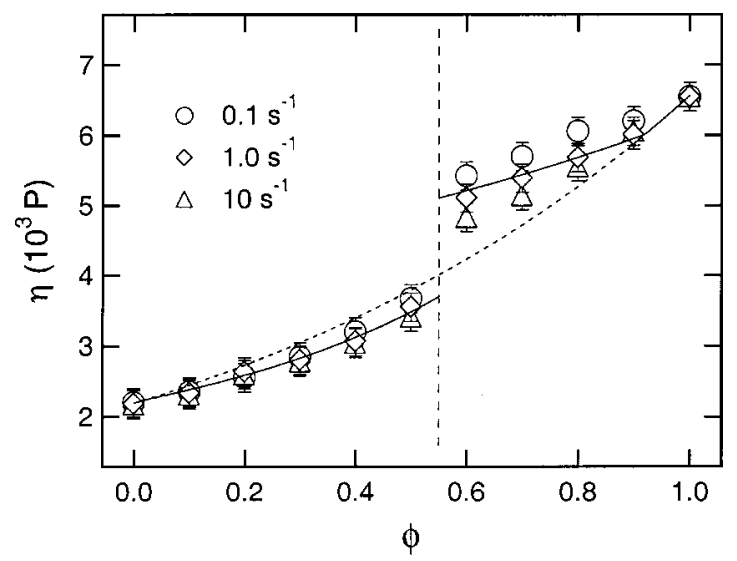

FIG. 1. Shear viscosity of the blends for different shear rates as a function of the PI volume fraction, where the dashed curve is the viscosity of the homogenized mixture, assuming log-additivity of the melt viscosities. The solid curve is a fit based on the simple physical model derived above. At $\phi=0.55$ (vertical dashed line), the system phase inverts from PI-rich droplets to PB-rich droplets, and the shear viscosity exhibits a discontinuity.

[1] H. van Oene, in Polymer Blends, edited by D. R. Paul and S. Newman (Academic Press, New York, 1978). 